\title{
An exact model for the multiplexing of worst case traffic sources ${ }^{1}$
}

\author{
J. García, J.M. Barceló, O. Casals \\ Polytechnic University of Catalonia \\ Computer Architecture Department \\ c/ Gran Capitán, Módulo D6 \\ E-08071 Barcelona, Spain \\ tel $:+3434015956$ \\ fax $:+3434017055$ \\ e-mail : (jorge,joseb,olga)@ac.upc.es
}

\begin{abstract}
In this paper we analyse a multiplexer handling a number of identical and independent Worst Case Traffic (WCT) sources. Each WCT source produces a periodic stream of cells consisting of a constant number of back-to-back cells followed by a silent period of constant duration. The WCT can model the traffic produced by a "malicious" user who sends an ON/OFF traffic where a burst of back-to-back cells whose length is the largest compatible with the tolerance introduced in the control function alternates with an idle period whose length is the smallest compatible with the policed peak cell rate. WCT can also model, for example, the traffic produced by some ATM Adaptation Layer multiplexing schemes in the Terminal Equipment.

Exact results are obtained, both for the discrete and the fluid-flow model. The numerical examples show the dramatic impact that WCT can have on the multiplexer buffer requeriments. The model presented can be useful to assess the convenience of using a traffic shaping device at the entry point of the ATM network.
\end{abstract}

\section{Keywords}

Worst Case Traffic, ATM, Traffic Management, Congestion Control.

\footnotetext{
${ }^{1}$ This work was supported in part by the CIRIT, Grant no. GRQ93-3.008
} 


\section{INTRODUCTION}

The B-ISDN which will be based on the ATM technique, is designed to transport a wide variety of traffic classes with different transfer capacity needs and Network Performance objectives. The traffic flow present in such networks will be subject to unpredictable statistical fluctuations which will cause congestion. During a congestion state, the network will not be able to meet the negociated Network Performance objectives for the already established connections.

It is generally assumed that for real time services Traffic Control for ATM networks will be done in three steps:

- The user requests the set up of a connection characterized by a declared Traffic Descriptor.

- The network determines by means of a Connection Admission Control (CAC) function whether this connection can be accepted while maintaining the agreed Quality of Service (QoS). If the connection can be accepted, the network allocates the necessary resources.

- The network controls the established connection by means of a User Parameter Control (UPC) algorithm to verify that the negociated parameters of the Traffic Contract are not violated.

The Traffic Contract at the Public UNI consists of a Connection Traffic Descriptor, a requested QoS class and the definition of a compliant connection The ATMForum, 1993. The Connection Traffic Descriptor consists of:

- The Source Traffic Descriptor which can include parameters like Peak Cell Rate, Sustainable Cell Rate, Burst Tolerance and/or source type.

- The Cell Delay Variation (CDV) Tolerance. CDV refers to the random perturbation on the interarrival time of consecutive cells of a given connection produced by cell multiplexing and other functions of the ATM Layer. CDV Tolerance represents a bound on the cell clumping phenomenon due to CDV and it is defined according to the UPC algorithm used.

- The Conformance Definition based on one or more applications of the Generic Cell Rate Algorithm (GCRA). The GCRA is a Virtual Scheduling Algorithm or 
a Continuous-state Leaky Bucket Algorithm which is used to specify the conformance at the public or private UNI to declared values of CDV tolerance and of traffic parameters Peak Cell Rate, Sustainable Cell Rate and Burst Tolerance.

An important consequence of the introduction of a CDV Tolerance is that the UPC algorithm will allow that a certain number of cells violate the Peak Cell Rate declared at connection set up. In fact this tolerance makes possible that a burst of a certain number of back-to-back cells (i.e. emitted at link rate) are viewed as conforming cells by the UPC algorithm.

As an example, let us assume that at connection set up a user declares a Constant Bit Rate (CBR) connection with a certain Peak Cell Rate and a certain CDV Tolerance. The UPC algorithm will declare as conforming a maximum of $N$ back-to-back cells if this burst of cells is preceded by a silence state long enough. In fact the user could send a periodic flow of cells consisting of $N$ back-to-back cells followed by a silence state and the UPC will declare this flow of cells as conforming. We will call this kind of periodic traffic Worst Case Traffic (WCT). This name comes from the fact that the multiplexing of this kind of traffic requires by far more resources than the required by a periodic connection. In fact WCT is not the "worst" traffic that can be declared as conforming by the UPC algorithm (see Aarstad, 1993). However WCT as defined above is more tractable and the results obtained are not far from the "worst" case.

The study of the effect of WCT in ATM multiplexers is important for several reasons: A misbehaving customer can try to take advantage of the UPC function tolerance to send traffic with different parameters than the negociated during the Connection set up phase. In our example the network cannot relay on the assumption that the user is actually sending CBR traffic as declared because it does not have means of checking that this is really happening. Therefore in order to ensure a certain QoS the CAC has to assume WCT to decide whether a connection can be accepted or not.

Besides the "tricky user" some AAL schemes may generate WCT in a natural way. As an example (Boyer, 1992), let us assume that a multimedia workstation involves several AALs each of them generating CBR traffic at different peak bit rates. Each AAL accumulates data in a private queue. The queues are periodically emptied at a rate of 150 Mbps with scanning period set to the lowest involved peak emission period.

A possible solution to the problem caused by clumps of cells is to use a Traffic Shaping function together with the UPC/NPC in order to retrieve as far as possible the negociated Peak Cell Rate of the connection. A device which performs this shaping function (together with a UPC function) known as Spacer/Controller has been proposed (see Boyer, 1992, Wallmeier, 1992). The model presented in the paper can be used to study the trade-off between the cost of introducing a shaping device such as the Spacer/Controller and the 
low utilization or large buffers needed to cope with WCT.

In this paper we present an exact model for the multiplexing of $N$ identical and independent WCT sources. In García an approximate model for the multiplexing of WCT together with geometric or VBR traffic is developed. Approximate solutions of the model presented here are found in Roberts, 1993 (fluid approach) and in Kvols, 1992 (discrete time). In both cases the authors use what is known as the Benes bound which gives an upper bound for the queue length distribution. The problem of obtaining the queue length distribution using the Benes approach which can be reduced to computing the $i$ th-fold convolution of a pulse, is solved approximately in Roberts, 1993 by using the saddle-point method and in Kvols, 1992 by means of an FFT for the discrete time case. In appendix A and B we give closed formulas for these convolutions for both the discrete and fluid case. In Ramamurthy, 1991 an exact formula for the mean queue length is given and they suggest an approximation for the queue length distribution.

The model developed is used to demonstrate the decrease of network utilization when considering WCT or, alternatively, the increase of buffer length needed to maintain a certain QoS. Roughly, allowing clumps of length $b$ in the network means to increase the required buffer capacity by a factor of $b$ if no traffic shaping is used.

The paper is structured as follows: In section 2 we present a discrete time model of the multiplexing of $N$ identical and independent WCT sources in a slotted queue. In section 3 we see how to extend the model for a fluid approach. In section 5 we compare the exact results we obtain with some approximation suggested in the literature. In section 5 we use the model developed to study the impact of WCT in the dimensioning of ATM networks. Finally conclusions are drawn in section 6 .

\section{MULTIPLEXING WORST CASE TRAFFIC SOURCES IN A SLOT- TED QUEUE}

In this section we consider a multiplexer of capacity one cell per time slot loaded with $N$ identical and independent Worst Case Traffic (WCT) sources. Each WCT source produces a periodic stream of cells, of period $T$, with the following pattern: It emits a constant number, $b$, of back-to-back cells and then it remains silent during a constant time $T-b$ (Figure 1). The time slots where each source becomes active are uniformly and independently distributed within the period. In order to have a stable queue we assume that $\frac{N b}{T}<1$. We consider that arrivals take precedence on departures (i.e. first we have cell arrivals (if any), then the service (if any), and finally we observe the system).

We use the following definitions: $N(t)$ is the number of arrivals at slots $-(t-1), \ldots, 0$; $\phi(t)=N(t)-t, B_{t}$ is the number of sources which become active at slots $-(t-1), \ldots, 0$; 


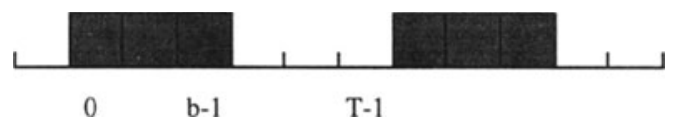

Figure 1: Traffic offered by a WCT source

$\gamma_{t}$ is the number of sources which were active at $-t$, and $L_{t}$ is the queue length at $-t$.

Following the Benes approach for the analysis of a slotted statistical multiplexer with a periodic input of period T (see Roberts, 1991) we express $p\left\{L_{0}>x\right\}$ as:

$$
p\left\{L_{0}>x\right\}=\sum_{t=1}^{T} p\{\phi(t)=x\} p\{\phi(u)<x, t<u \leq T \mid \phi(t)=x\} .
$$

Introducing $\gamma_{t}$ and $B_{t},(1)$ can be written as:

$$
\begin{aligned}
& p\left\{L_{0}>x\right\}= \\
& \sum_{t=1}^{T} \sum_{i=1}^{N} p\left\{\phi(t)=x, \gamma_{t}=0, B_{t}=i\right\} p\left\{\phi(u)<x, t<u \leq T \mid \phi(t)=x, \gamma_{t}=0, B_{t}=i\right\} .
\end{aligned}
$$

2.1 The term $p\left\{\phi(t)=x, \gamma_{t}=0, B_{t}=i\right\}$.

The term $p\left\{\phi(t)=x, \gamma_{t}=0, B_{t}=i\right\}$ can be expressed as

$$
p\left\{\phi(t)=x, \gamma_{t}=0, B_{t}=i\right\}=p\left\{\phi(t)=x \mid \gamma_{t}=0, B_{t}=i\right\} p\left\{\gamma_{t}=0, B_{t}=i\right\} .
$$

To derive an expression for these probabilities, we distinguish between three cases depending on the values of $t$ :

- Case $(I): t=1, \ldots, b-1$

We have:

$$
p\left\{\gamma_{t}=0, B_{t}=i\right\}=\left(\begin{array}{c}
N \\
i
\end{array}\right) \frac{t^{i}(T-b-t)^{N-i}}{T^{N}} .
$$

and:

$$
p\left\{\phi(t)=x \mid \gamma_{t}=0, B_{t}=i\right\}=\frac{1}{t^{i}} q_{t}^{(i)}(t+x),
$$

where $q_{t}(x)$ is a discrete-time unitary pulse in $[1, t]$ and $q_{t}^{(i)}(x)$ is its $i$-th discrete-time convolution. A simple expression for $q_{t}^{(i)}(x)$ is derived in Appendix A. 
- Case (II): $t=b, \ldots, T-b$

$p\left\{\gamma_{t}=0, B_{t}=i\right\}$ has the same expression as in case (I) (equation (3)). For the other term we get:

$$
p\left\{\phi(t)=x \mid \gamma_{t}=0, B_{t}=i\right\}=\sum_{j=0}^{i}\left(\begin{array}{c}
i \\
j
\end{array}\right) \frac{(t-b+1)^{j}}{t^{i}} q_{b-1}^{(i-j)}(t+x-b j) .
$$

- Case (III): $t=T-b+1, \ldots, T$

For $i=1, \ldots, N-1$ this term vanishes. For $i=N$ we have:

$$
p\left\{\gamma_{t}=0, B_{t}=N\right\}=\left(\frac{t}{T}\right)^{N} .
$$

and

$$
p\left\{\phi(t)=x \mid \gamma_{t}=0, B_{t}=N\right\}=\sum_{j=0}^{N}\left(\begin{array}{c}
N \\
j
\end{array}\right) \frac{(t-b+1)^{j}}{t^{N}} q_{b-1}^{(N-j)}(t+x-b j) .
$$

2.2 The term $p\left\{\phi(u)<x, t<u \leq T \mid \phi(t)=x, \gamma_{t}=0, B_{t}=i\right\}$.

This term can be written as:

$$
\begin{aligned}
p\{\phi(u) & \left.<x, t<u \leq T \mid \phi(t)=x, \gamma_{t}=0, B_{t}=i\right\}= \\
& =\frac{p\left\{\phi(u)<x, t<u \leq T \mid \phi(t)=x, B_{t}=i\right\}}{p\left\{\gamma_{t}=0 \mid \phi(t)=x, B_{t}=i\right\}}
\end{aligned}
$$

A simple expression for the numerator can be derived by means of a similar argument as in section III of Roberts, 1991:

We note that the event $\left\{\phi(u)<x, t<u \leq T \mid \phi(t)=x, B_{t}=i\right\}$ corresponds to the arrival patterns that would result in an auxiliar queue loaded with periodic arrivals of period $T-t$ being empty at time $-t$.

The periodic arrivals at this auxiliar queue belong to two classes (Figure 2):

- A batch arrival at time $-(T-1)$ consisting of $i b-t-x$ cells. These cells correspond to the ones that were emitted in the original system by the $i$ sources that had become active at slots $-(t-1-T), \ldots,-T$.

- $N-i$ independent WCT sources of period $T-t$ emitting $b$ back-to-back cells. 
If the original system is stable, the same will occur with the auxiliar queue $(N b<T$ implies $i b-t-x+(N-i) b<T-t)$. Therefore we do not need to take into account the contribution of the first batch arrival, obtaining:

$$
p\left\{\phi(u)<x, t<u \leq T \mid \phi(t)=x, B_{t}=i\right\}=1-\frac{(N-i) b}{T-t} .
$$

The denominator is : $p\left\{\gamma_{t}=0 \mid \phi(t)=x, B_{t}=i\right\}=\left(\frac{T-t-b}{T-t}\right)^{N-i}$.

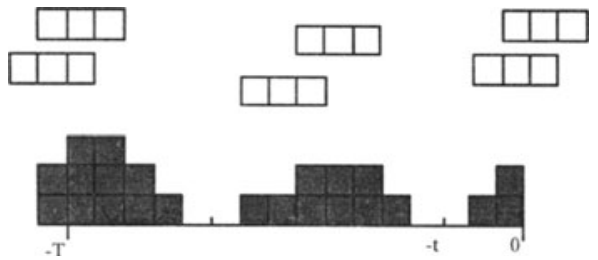

(a) Original system (Period $=\mathrm{T})$

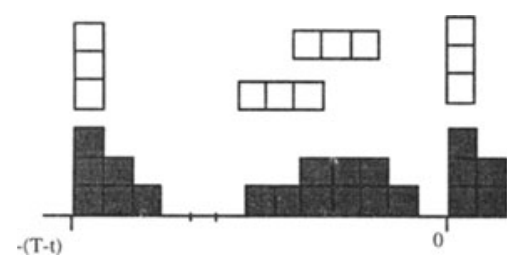

(b) Equiv. system with batch arrival at first slot (Period $=\mathrm{T}-\mathrm{t})$

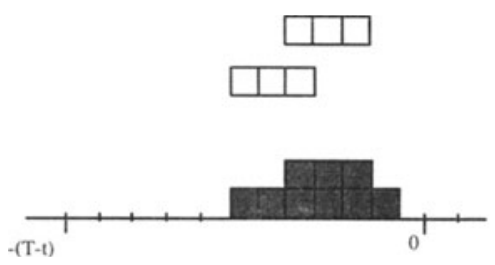

(c) Equiv. system without batch arrival at first slot (Period $=\mathrm{T}-\mathrm{t})$

Figure 2:

\section{THE FLUID WCT MODEL}

A similar study can be done for a system using a fluid-flow approximation. In this case the WCT is defined as a periodic source which produces $h$ information units per time slot during a constant time $b$ and which remains silent during a constant time $T-b$. The 
multiplexer is able to serve at rate $c$. We asume $h \geq c$. The analogous to (1) in the fluid case is (see Roberts, 1993):

$$
p\left\{L_{0}>x\right\}=c \int_{0}^{T} p(\psi(t)=x) p(\psi(u)<x, t<u \leq T \mid \psi(t)=x\} d t
$$

where $W(t)$ is the work arriving in the interval $(-t, 0)$, and $\psi(t)=W(t)-c t$.

The derivation of the final formula in the case of WCT fluid sources follows the same steps as in the discrete-time case: We also have to distinguish between three cases depending on the values of $t$. (Namely: case (I): $\mathrm{t}<\mathrm{b}$; case (II): $\mathrm{b}<\mathrm{t}<\mathrm{T}-\mathrm{b}$ and case (III): $\mathrm{T}-\mathrm{b}<\mathrm{t}$ ). The formula for the $i$-th convolution of a continuous-time pulse is derived in appendix B. The resulting integrals can be evaluated, for example, by means of the Gauss method.

\section{ERRORS AND BOUNDS}

It is well known that the queue length distribution of a $n D / D / 1$ queueing system can be approximated by the queue length distribution of an $M / D / 1$ queueing system when the period of the sources is large (see Roberts, 1991). In Figure 3 we make a comparison between the $n W C T / D / 1$ system and a slotted queue loaded with Poisson batch arrivals (curve with the points). The batchs have a deterministic distribution of $b=2$ cells. We observe that when the period becomes large, the two systems have a similar distribution. The queue with Poisson batch arrivals gives an upper bound of the buffer length distribution of the $n W C T / D / 1$ system.

In Ramamurthy, 1991 the following approximation is suggested: Let $L^{(b)}$ be the queue length of a multiplexer loaded with $N$ WCT sources of period $T^{\prime}=b T$ for a given $T$. Then

$$
p\left\{L^{(b)}>x\right\} \approx p\left\{L^{(1)}>\frac{x}{b}\right\} .
$$

Figure 4 compares this approximation (curves with the points) with the results obtained from the exact model. As expected, the differences with the exact model are more important for larger values of $b$.

\section{RESULTS}

In this section we present some results obtained for the discrete-time case. 


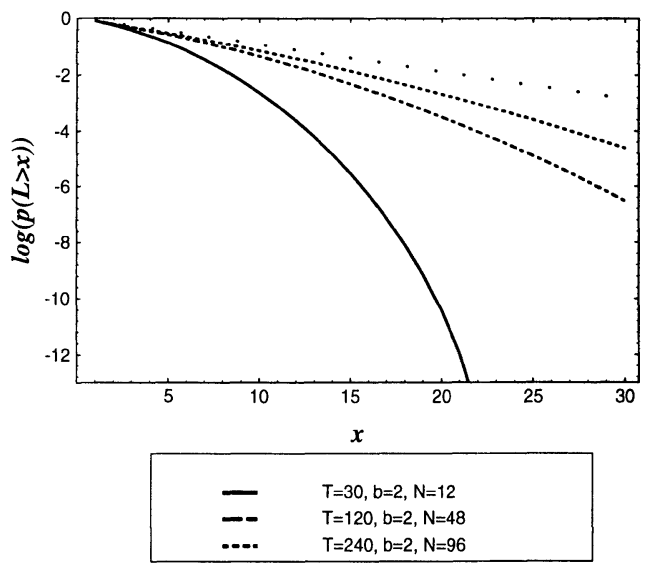

Figure 3:

Figure 5 shows, for different values of $b$, the complementary queue length distribution of a multiplexer handling 12 WCT traffic. The ratio $\frac{b}{T}=\frac{1}{15}$ is maintained constant. Even for $b=2$ and $b=3$ we observe a dramatic impact on the queue length distribution: For example, if we have CBR connections $(b=1)$ the probability of having more than 10 cells in the multiplexer is $3.277 \times 10^{-11}$. In the case of WCT with $b=2$ the probability of this event is incremented about 6 orders of magnitude $\left(7.823 \times 10^{-4}\right)$ while with $b=3$ we obtain a value of $1.737 \times 10^{-2}$. On the other hand, to have a quantile of the buffer length probability lower than $1 \times 10^{-10}$ we need for $b=1$ a buffer length $L=10$, for $b=2$ we need $L=20$ while for $b=3, L=28$.

Figure 6 shows the admissible load to have a quantile of the buffer queue length probability lower than $1 \times 10^{-10}$. The traffic parameters are the same as above. We can again observe the impact of the value of $b$ : For $b=1$ the admissible load is 0.80 while for $b=2$ it decreases to 0.33 and for $b=3$ to 0.27 .

A similar experiment is performed when $\frac{b}{T}=\frac{1}{100}$. We observe again the strong impact of $b$ on the complementary buffer length distribution for a multiplexer loaded up to 0.8 (figure 7) and on the admissible load when the buffer has a capacity of 24 cells (figure 8).

Now we study an example in which the user produces WCT. Let us assume that we scan each 480 time slots the AAL buffers of a multimedia workstation with different CBR connections. The user generates traffic at a total rate of $10 \mathrm{Mbps}$, and the physical link rate is $150 \mathrm{Mbps}$. This means that clumps of 32 back-to-back cells will enter the ATM network. Figure 9 shows the complementary buffer queue length distribution of a multiplexer handling such traffic sources (load 0.80). To have a quantile of the buffer length 


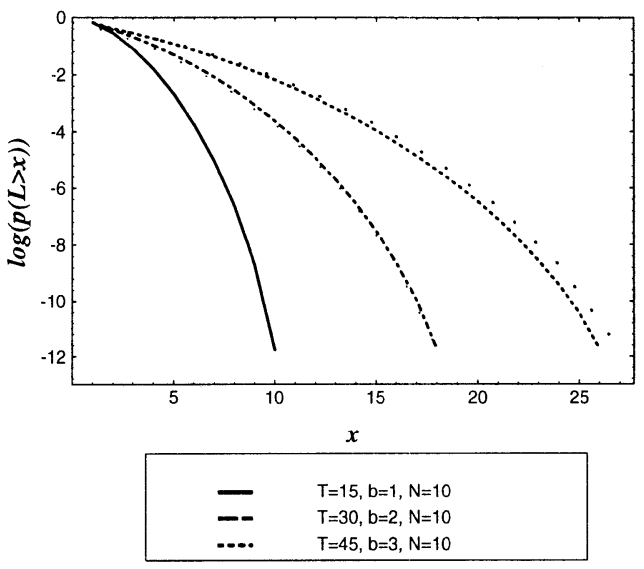

Figure 4:

probability lower than $1 \times 10^{-10}$ we need a value of $L=288$. Using $L=128$ means to reduce the load to 0.33 while for $L=64$ the admissible load is 0.13 .

\section{CONCLUSIONS}

In this paper we have developed an exact model for a multiplexer loaded with a number of identical and independent Worst Case Traffic (WCT) sources.

The model can be used to demonstrate the decrease of network utilization when considering WCT or, alternatively, the increase of buffer length needed to maintain a certain QoS. This analysis is important to assess the convenience of using traffic shaping devices at the ATM entry points.

\section{ACKNOWLEGDMENT}

We would like to thank Pierre Boyer for useful discussions and suggestions.

\section{APPENDIX A : I-TH CONVOLUTION OF A PULSE IN DISCRETE TIME}

Let $q_{m}(t)$ be a discrete-time pulse of amplitude 1 in $[1, m]$ and let $q_{m}^{*}(z)$ be the z-transform of such pulse. We are interested in finding a simple expression for the $i$-th discrete-time convolution of $q_{m}(t)$. This is equivalent to find a simple expression for the coefficients of 


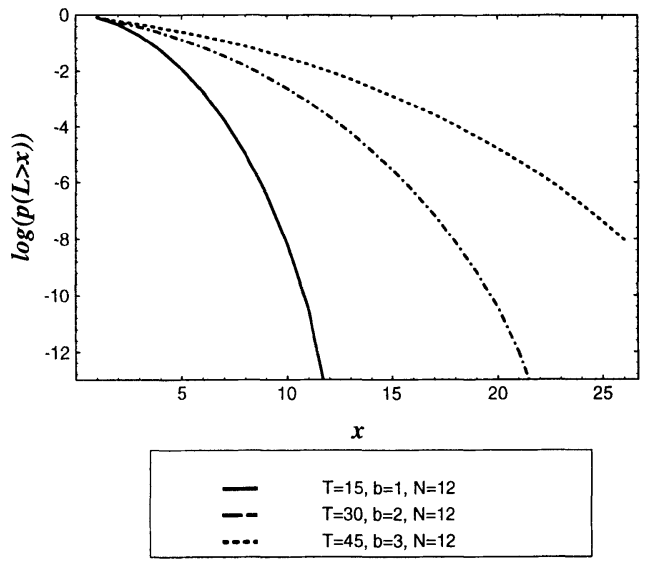

Figure 5:

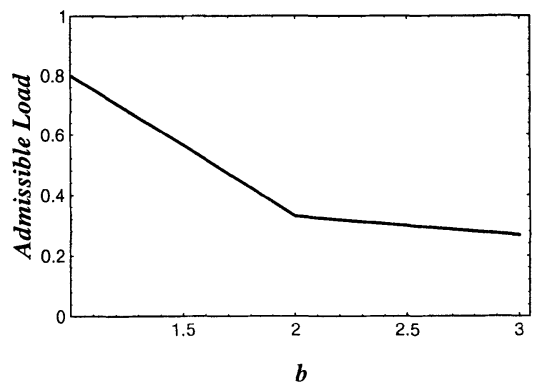

Figure 6:

the polynomial:

$$
\left(q_{m}^{*}(z)\right)^{i}=\left(\sum_{n=1}^{m} z^{n}\right)^{i} .
$$

We can express $q_{m}^{*}(z)$ as:

$$
q_{m}^{*}(z)=\left(1-z^{m}\right) p_{\infty}^{*}(z) .
$$

We can easily derive (from example, using the convolution algorithm, Buzen, 1973 that:

$$
\left(q_{\infty}^{*}(z)\right)^{i}=z^{i} \sum_{n=0}^{\infty}\left(\begin{array}{c}
i+n-1 \\
n
\end{array}\right) z^{n} .
$$




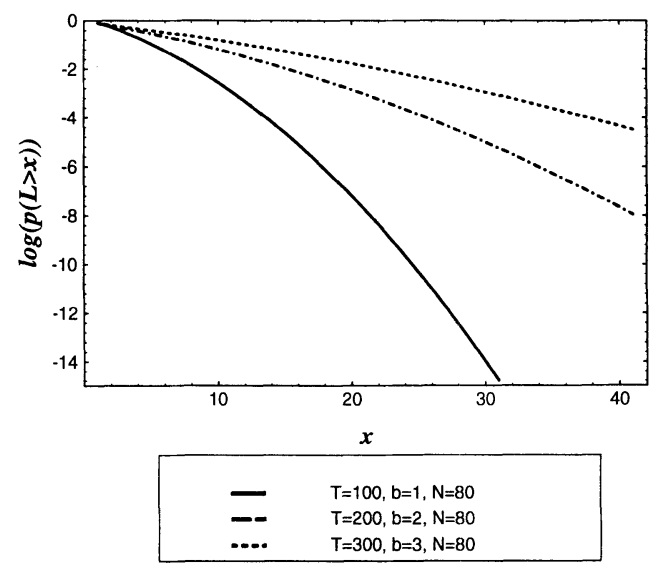

Figure 7:

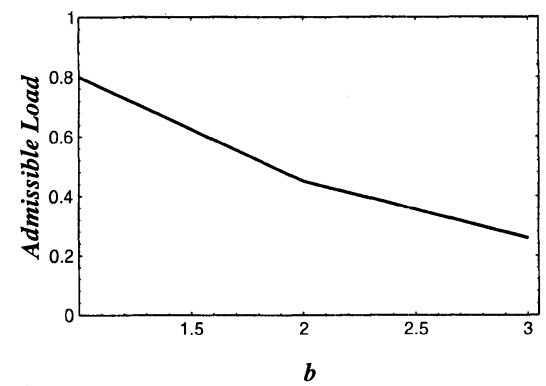

Figure 8:

Hence, we obtain $(i>0)$ :

$$
\left(q_{m}^{*}(z)\right)^{i}=z^{i} \sum_{n=0}^{i(m-1)} \sum_{s=0}^{\left[\frac{n}{m}\right]}(-1)^{s}\left(\begin{array}{c}
i+n-s m-1 \\
n-s m
\end{array}\right)\left(\begin{array}{l}
i \\
s
\end{array}\right) z^{n} .
$$

From that we deerive a formula for $q_{m}^{(i)}(t)(i>0$ and $t=i, \ldots, i m)$ :

$$
q_{m}^{(i)}(t)=\sum_{s=0}^{\left[\frac{t-i}{m}\right]}(-1)^{s}\left(\begin{array}{c}
i \\
s
\end{array}\right)\left(\begin{array}{c}
t-s m-1 \\
i-1
\end{array}\right) .
$$

and $q_{m}^{(i)}(t)=0$ for other values of $t$.

For $i=0$ we define

$$
q_{m}^{(0)}(t)=\delta(t)
$$




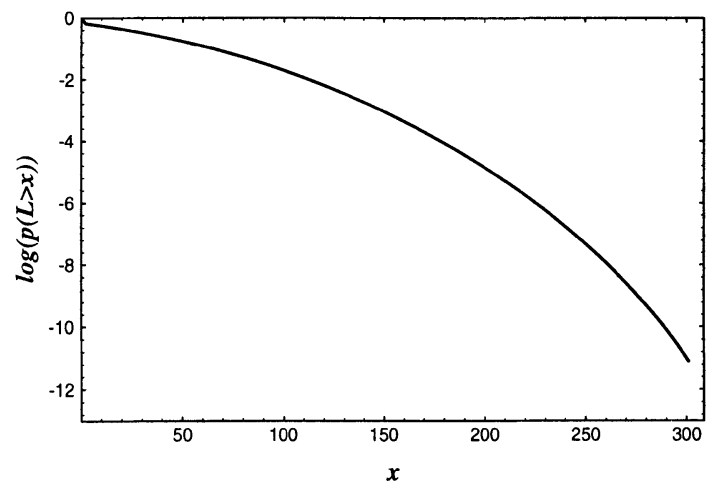

Figure 9:

\section{APPENDIX B : I-TH CONVOLUTION IN CONTINUOUS TIME}

Let $p_{y}(x)$ be a pulse with unitary amplitude in $(0, y)$. In order to get an explicit formula for its $i$-th convolution, we obtain first the Fourier transform of $p_{y}(x)$ :

$$
P_{y}(\omega)=\int_{-\infty}^{+\infty} e^{-j \omega x} p_{y}(x) d x=j \frac{e^{-j \omega y}-1}{\omega} .
$$

Hence, the transform of $p_{y}^{(i)}(x)$ is:

$$
P_{y}^{i}(\omega)=j^{i}\left(\frac{e^{-j \omega y}-1}{\omega}\right)^{i}
$$

and $p_{y}^{(i)}(x)$ can be expressed as $(i>0)$ :

$$
p_{y}^{(i)}(x)=\frac{1}{2 \pi} \int_{-\infty}^{+\infty} e^{j \omega x} P_{y}^{i}(\omega) d \omega=\frac{j^{i}}{2 \pi} \sum_{k=0}^{i}\left(\begin{array}{c}
i \\
k
\end{array}\right)(-1)^{i-k} \int_{-\infty}^{+\infty} \frac{e^{-j \omega(k y-x)}}{\omega^{i}} d \omega .
$$

We now consider the integral:

$$
\int_{-\infty}^{+\infty} \frac{e^{j \omega(x-k y)}}{\omega^{i}} d \omega=\int_{-\infty}^{+\infty} \frac{\cos (\omega(x-k y))}{\omega^{i}} d \omega+j \int_{-\infty}^{+\infty} \frac{\sin (\omega(x-k y))}{\omega^{i}} d \omega
$$

If $i$ is even the imaginary part of the rigth hand side of equation (20) vanishes. For the real part we have Papoulis, 1962, Prudnikov): 


$$
\begin{aligned}
\int_{-\infty}^{+\infty} \frac{\cos (\omega(x-k y))}{\omega^{i}} d \omega & =\frac{(x-k y)^{i-1}}{(i-1) !} \cos \left(i \frac{\pi}{2}\right) \int_{-\infty}^{+\infty} \frac{\sin (\omega(x-k y))}{\omega} d \omega= \\
& =\pi \frac{|x-k y|^{i-1}}{(i-1) !} \cos \left(i \frac{\pi}{2}\right)
\end{aligned}
$$

If $i$ is odd the real part vanishes while for the imaginary part we have:

$$
\begin{aligned}
\int_{-\infty}^{+\infty} \frac{\sin (\omega(x-k y))}{\omega^{i}} d \omega & =\frac{(x-k y)^{i-1}}{(i-1) !} \cos \left((i-1) \frac{\pi}{2}\right) \int_{-\infty}^{+\infty} \frac{\sin (\omega(x-k y))}{\omega} d \omega= \\
& =\pi \frac{(x-k y)^{i-1}}{(i-1) !} \cos \left((i-1) \frac{\pi}{2}\right) \operatorname{sgn}(x-k y) .
\end{aligned}
$$

Finally we obtain the following expression for $p_{y}^{(i)}(x)(i>0)$ :

$$
p_{y}^{(i)}(x)=\frac{1}{2} \sum_{k=0}^{i}\left(\begin{array}{c}
i \\
k
\end{array}\right)(-1)^{k} \frac{(x-k y)^{i-1} \operatorname{sgn}(x-k y)}{(i-1) !} .
$$

For $i=0$ we define:

$$
p_{y}^{(0)}(x)=\delta(x)
$$

where $\delta(x)$ denotes the Dirac delta in continuous-time.

\section{REFERENCES}

Aarstad E. A comment on Worst Case Traffic, COST 242 TD, 1993.

Boyer, P. Guillemin F. M., Servel M. J. and Coudreuse J. P. Spacing Cells At The ATM Network Entry Points. Special Issue of IEEE Network Magazine on Switching and Congestion Control in ATM Networks, September 1992.

Buzen J. P. Computational Algorithm for Closed Networks with Exponential Servers", Comm. Assoc. Comput. Mach., 1973, 16, 527-531.

García J., Casals O, A Discrete Time Queueing Model to Study the Cell Delay Variation in an ATM Network", Performance Evaluation 21 3-22, 1994.

Kvols K., Blaabjerg S. Bounds and Approximations for the Periodic On/Off Queue with Applications to ATM Traffic Control, IEEE Infocom'92, Florence, May 1992.

Papoulis A. The Fourier Integral and its Applications, McGraw-Hill, 1962, New York. Prudnikov A. P., Brychkov Y. A. and Marichev O. I. Integrals and Series, Volume 1. Gordon and Breaach Science Publishers, New York. 
Ramamurthy G., Dighe R. S. A Distributed Source Control: A Network Access Control for Integrated Broadband Packet Networks. IEEE JSAC, vol. 9, No. 7, pp 990-1002, September 1991.

Roberts J. W., Virtamo J. The Superposition of Periodic Cell Arrival Streams in an ATM Multiplexer, IEEE T. on Comm., Vol. 39, No. 2, Feb. 1991.

Roberts J. W., Bensaou B., Canetti Y. A Traffic Control Framework for High-Speed Data Transmission, Modelling and Performance Evaluation of ATM Technology, La Martinique, January 1993.

The ATM Forum, ATM User-Network Interface Specification, Version 3.0, September, 1993.

Wallmeier E., Worster T. The Spacing Policer, an Algorithm for Efficient Peak Bit Rate Control in ATM Networks, Proc. of ISS'93, Yokohama (Japan), October 1992.

\section{BIOGRAPHY}

Jorge García graduated and received his Ph.D. in Telecommunications Engineering from Polytechnic University of Catalonia (UPC) in 1988 and 1992, respectively. He joined UPC in 1988 and currently is Associate Professor of the Computer Architecture Department. In 1992-93 he was Visiting Scientist at the Systems and Industrial Department of the University of Arizona with a NATO fellowship. He has ben involved in several RACE project and currently is involved in COST-242 project.

José María Barceló graduated in Telecommunications Engineering from UPC in 1992. He has been involved in RACE project EXPLOIT and currently he is Ph. D. student in the Computer Architecture Department of UPC.

Olga Casals graduated and received his Ph. D. from UPC in 1983 and 1986 respectively, both in Telecommunications Engineering. She joined UPC in 1983 where she became Full Professor in 1994 and she is head of a research group on traffic in B-ISDN communications systems. She has been working on ATM networks since 1988 with her participation in the RACE project R1022. She has been also involved in the RACE projects EXPLOIT and BAF and currently she is involved in ACTS project EXPERT and in COST-242. 